\title{
Deux nouvelles parutions sur le Ménexène de Platon par Étienne HELMER et David SANSONE
}

PLATON, Ménexène, introduction, nouvelle traduction (texte grec en regard) et commentaire par Étienne HELMER \& PLATON, Menexenus, edited by David SANSONE

\section{Thomas Bénatouïl}

\section{(2) OpenEdition}

\section{Journals}

Édition électronique

URL : https://journals.openedition.org/philosant/4258

DOI : $10.4000 /$ philosant.4258

ISSN : 2648-2789

Éditeur

Éditions Vrin

\section{Édition imprimée}

Date de publication : 15 décembre 2021

Pagination : 273-275

ISBN : 978-2-7116-3036-3

ISSN : 1634-4561

\section{Référence électronique}

Thomas Bénatouïl, « deux nouvelles parutions sur le Ménexène de Platon par Étienne HeLmer et David SANSONE », Philosophie antique [En ligne], 21 | 2021, mis en ligne le 28 mai 2021, consulté le 06 décembre 2022. URL : http://journals.openedition.org/philosant/4258 ; DOI : https://doi.org/10.4000/ philosant.4258

\section{(c) $($ ) $९$ EY}

Creative Commons - Attribution - Pas d'Utilisation Commerciale - Pas de Modification 4.0 International - CC BY-NC-ND 4.0

https://creativecommons.org/licenses/by-nc-nd/4.0/ 
be fixed by anything (or if they are fixed by something, we need to know by what). And then Aristotle is right, that nothing has been explained, if something makes them always equal but we are not told what.

To this puzzle, which underpins my own radically different proposal - that these periods are not set, nor need they be equal, from cycle to cycle, though they are always symmetrical and equal within a cycle - the Byzantine scholia offer no kind of solution. Rashed's scholia give inexplicable numbers that cannot answer Aristotle's question, whichever equality Aristotle was talking about. Producing some mystical numbers that add up to a multiple of 10,000 years is not providing a reason for that regularity, but merely asserting an arbitrary regularity, which strikes me as deeply unsatisfying. Why did Aristotle not discover Empedocles' properly satisfying answer to the questions about what sets the durations? Because, I would hazard, he looked primarily among the material to which we have conventionally attached the name "physics", and because in reality no answer to that question about durations can be supplied in physics, because the question about durations is (I think) not a question about what we think of as physics, and the basis for an answer is not to be found in the "physics" material. I suspect that is also why no one else, among those whom Rashed calls the "literal readers" of the cycle, could give an answer either, if they too (as Rashed does here) segregate the material related to ethics and the agency of daimones, and assign it to a separate story, in a separate poem called Katharmoi. This is where I differ from Rashed (as well as many others) who assume that the question about how long each section of the cosmic cycle lasts needs a mechanistic answer, rather than (as I would suggest) an ethical answer. That is, if the duration is variable, and is set by voluntary actions and their consequences, then the answers to "why?" and "how long?" have more to do with ethics than with the behaviour of inert elements.

Catherine RowetT

University of East Anglia, Norwich, UK

Platon, Ménexène, introduction, nouvelle traduction (texte grec en regard) et commentaire par Étienne Helmer, Paris, Vrin, 2019 (Les dialogues de Platon, 2), 183 p., ISBN : 978-2-7116-2826-1

Platon, Menexenus, edited by David Sansone, Cambridge, Cambridge University Press, 2020 (Cambridge Greek and Latin Classics), x +193 p. ISBN : 978-1-108-73056-3.

À l'instar de nombreux dialogues socratiques de Platon, le Ménexène s'ouvre sur une rencontre fortuite, près de l'agora, entre Socrate et le jeune Ménexène (qui est présent dans le Lysis et aussi dans le Phédon). Ils engagent une discussion à propos de l'éloge des soldats morts au combat traditionnellement prononcé lors de leurs funérailles publiques à Athènes, qui doivent bientôt avoir lieu et pour lesquelles l'orateur doit être choisi par le Conseil. Socrate estime que ce type de discours n'est pas difficile à composer et, face à la surprise de Ménexène, finit par proposer lui-même un exemple d'un tel éloge, qui aurait été écrit par Aspasie, auprès de qui Socrate dit apprendre la rhétorique, comme l'avait déjà fait Périclès. Suit l'oraison funèbre d'Aspasie-Socrate, qui occupe les quatre cinquièmes de ce court texte et qui suscite pour finir l'approbation et la reconnaissance de Ménexène... et le trouble ou le désintérêt de nombreux lecteurs de Platon, en particulier des philosophes, qui ont très peu fréquenté ce « dialogue ».

Les deux ouvrages recensés ici sont similaires par la taille, le public visé (plutôt des étudiants) et la structure (une introduction, le texte grec, un commentaire suivi, 
une bibliographie) mais se distinguent en fonction des exigences des collections auxquelles ils appartiennent. L'ouvrage d'Étienne Helmer (EH), qui est le second d'une collection récente (inaugurée par une traduction commentée du Politique publiée en 2018), comprend le texte grec de Burnet sans apparat critique et, en regard, une traduction française nouvelle du texte. Son commentaire se concentre sur la dimension philosophique du texte. L'ouvrage de David Sansone (DS) s'inscrit dans une collection née il y a cinquante ans et qui comprend près de cent volumes reconnaissables à leur couleur (vert et jaune) : il n'inclut pas de traduction mais seulement une édition du texte grec avec un apparat minimal (qui s’appuie sur les éditions les plus récentes), et son commentaire s'attache non seulement à l'interprétation philosophique du texte, mais aussi à sa dimension littéraire et à de nombreux points de grammaire et de traduction extrêmement utiles, notamment en ce qui concerne le style de Platon. La présente recension parallèle des deux ouvrages se concentrera sur les grands traits de la lecture qu'ils proposent du dialogue.

Comme le notent EH (p. 8) et DS (p. 37), les auteurs anciens comme Cicéron, le Ps.-Longin ou Denys d'Halicarnasse prennent l'oraison funèbre du Ménexène tout à fait au sérieux et n'hésitent pas à en citer certains passages comme exemplaires d'un point de vue rhétorique. Ce n'est qu'à la fin du XIX ${ }^{\mathrm{e}}$ siècle que le discours commence à être tenu pour parodique et donc ironique. L'interprétation non-ironique a toutefois retrouvé des défenseurs récemment, et le sens de cette oraison funèbre d'Aspasie rapportée par Socrate demeure donc très problématique. Platon semble avoir choisi de ne pas opposer de contre-analyse à ce discours non-philosophique, contrairement à ce qu'il fait dans le Gorgias (avec Calliclès), le Phèdre ou le Banquet. Cette stratégie originale du Ménexène a été bien analysée par Daniel Loayza à la fin de son introduction à sa traduction annotée (Platon, Ménexène, Paris, GF-Flammarion, 2006, p. 50-58). Elle n'est d'ailleurs peut-être pas unique ni réservée à la rhétorique chez Platon : dans la longue partie étymologique du Cratyle (391b-437d), Socrate se livre à des analyses qui imitent manifestement certaines spéculations de l'époque et ne les réfute pas ensuite, si bien que les commentateurs modernes divergent sur la question de savoir si elle est parodique ou si elle possède une valeur philosophique aux yeux de Platon.

Une première manière de traiter ce problème à propos de l'oraison funèbre de SocrateAspasie passe par la comparaison avec les autres oraisons funèbres que nous conservons, en particulier celle de Périclès reconstituée par Thucydide, mais aussi celles de Gorgias, Lysias ou Démosthène. C'est justement une approche de ce type qui a renouvelé l'étude du Ménexène et l'intérêt des spécialistes pour ce texte : celle de Nicole Loraux dans L'invention d'Athènes. Histoire de l'oraison funèbre dans la cité classique (publié en 1981) ainsi que dans plusieurs articles (que l'on retrouvera dans un tout récent et épais volume: N. Loraux, La Grèce hors d'elle et autres textes. Écrits 1973-2003, texte établi par Michèle Cohen-Halimi, Paris, Klincksieck, 2021). DS, qui souligne l'influence décisive de l'ouvrage de Nicole Loraux (p. ix), présente très utilement dans son introduction toutes les oraisons funèbres que nous avons conservées et propose ensuite des comparaisons toujours éclairantes du lexique, du style et des thèmes de l'oraison d'Aspasie-Socrate avec ceux des autres oraisons. Cela permet de saisir à quel point Platon suit de près et imite à la perfection les conventions de ce genre de discours.

Pour la partie de l'oraison qui célèbre les victoires militaires athéniennes, du combat contre les Amazones à la guerre de Corinthe (239a5-264a4), DS offre en outre des mises au point sur les autres récits disponibles, qui permettent de comprendre que Platon pousse à son extrême la réécriture nationaliste de l'histoire pratiquée par les orateurs (notamment Lysias); il procède par omission, sélection, brouillage ou recadrage 
des événements ou de leurs causes afin d'éluder tout ce qui pourrait ternir ou même amoindrir le prestige d'Athènes (voir par exemple p. 123-124 à propos de Marathon ou p. 143-144 à propos des défaites, toujours auto-infligées). La fin de ce passage pose d'ailleurs un problème important traité par DS dans son introduction (p. 12-17) : Aspasie-Socrate achève son histoire glorieuse d'Athènes par la guerre de Corinthe (395-386) qui est nettement postérieure à la mort de Socrate (399) et sans doute à celle d'Aspasie ( 400 ?). Alors que cet anachronisme a conduit certains interprètes à douter de l'authenticité du dialogue. DS estime à la suite d'autres interprètes récents qu'il est trop flagrant pour ne pas être délibéré et qu'il vise certainement à attirer l'attention des lecteurs sur l'indifférence à l'objectivité historique, sur la dimension fictionnelle et répétitive d'un tel éloge de la cité devant ses citoyens.

Une autre manière d'aborder l'éloge de Socrate-Aspasie consiste à s'appuyer sur des textes de Platon susceptibles d'expliciter les thèses sous-jacentes au Ménexène. Il s'agit d'abord des dialogues qui critiquent les pratiques des orateurs, le Gorgias, le Phèdre ou la République. EH les mobilise régulièrement pour souligner les limites ou méfaits de la rhétorique selon Platon et opposer la démarche de l'oraison funèbre d'Aspasie à celles du dialogue socratique voire de la dialectique platonicienne. Ce faisant, EH n'évite pas toujours le risque de reprocher à l'oraison funèbre de ne pas être un dialogue philosophique (p. 150) : peut-on par exemple s'étonner que l'oraison se réfère à des normes ou valeurs qu'elle n'explique pas (p. 107) ? S'il s'agit de dire que Ménexène devrait réclamer une telle justification dans le cadre de sa discussion privée avec Socrate afin de réaliser correctement ses ambitions politiques (234a-b), c'est tout à fait juste, mais une telle explication ne pourrait pas se faire dans le cadre d'une oraison funèbre, aussi parfaite soit-elle.

Beaucoup plus éclairantes de ce point de vue sont les comparaisons nombreuses qu'EH propose entre les propos de Socrate-Aspasie et des textes de la République, du Politique ou des Lois sur les valeurs et les pratiques civiques, soit que ces textes critiquent les valeurs et pratiques démocratiques athéniennes, soit qu'ils leur prescrivent une contrepartie dans une cité vertueuse, par exemple l'établissement d'un mythe d'autochtonie ou de rites funéraires pour les morts au combat (voir p. 84-87 sur République III, 414a-416b), soit qu'ils évoquent des faits historiques similaires, par exemple la nature de l'Empire perse et de la démocratie athénienne ou les rapports entre Grecs et Barbares (EH, p. 135-146).

Ces comparaisons systématiques conduisent $\mathrm{EH}$ à une interprétation d'ensemble du dialogue, qui vise à dépasser l'alternative entre le sérieux et la pure parodie : l'oraison funèbre d'Aspasie serait une « image dégradée » du discours politique inspiré par la philosophie (dont on trouve des esquisses dans la République ou les Lois), de même que la démocratie est « le résultat de la détérioration » du régime de la cité juste (p. 10-11, 80-81, 105-106 et 123-125). Une image dégradée n'étant pas une image simplement inversée, cette lecture a le grand intérêt de permettre de tenir compte à la fois des points communs et des différences entre l'oraison d'Aspasie et la politique philosophique (et de souligner, p. 14-15, qu'elles ne s'opposent pas non plus simplement comme une fiction à la vérité historique, puisque le philosophe fait également usage de fictions, comme en témoigne le Critias).

Peut-on pour autant affirmer que « l'objet [de l'oraison d'Aspasie] est de permettre au lecteur familier des Dialogues de Platon de mesurer l'écart séparant la parole politique démocratique et la parole politique fondée sur la philosophie » (p. 125) ? Les interprètes s'accordent (voir DS p. 14-15) pour dater le Ménexène de 386 environ et donc probablement d'une période où la République et le Critias, sans même parler du Politique et des Lois, ne sont pas écrits. Platon a donc mis par écrit « l'image dégradée » 
de la cité avant d'élaborer son modèle et, si la comparaison rétrospective des deux est légitime et souvent très éclairante, elle ne traduit vraisemblablement pas l'intention de Platon quand il a écrit le Ménexène.

Pour mieux saisir celle-ci, le court dialogue entre Socrate et Ménexène qui précède l'oraison funèbre et dont les lecteurs antiques ne semblent pas tenir compte, semble crucial. Il fait l'objet d'une analyse détaillée de la part de DS et EH. Socrate y décrit « l'ensorcellement » durable produit sur lui par les éloges des soldats morts qu'il a entendus, et nie qu'il soit difficile de préparer rapidement un éloge destiné à ceux dont il fait l'éloge (234e-235d). DS (p. 70-71) et EH (p. 84-85) montrent les parallèles de cette critique avec la comédie et avec les réflexions de Socrate sur l'éloge dans le Banquet (198d199a). Ménexène voit bien que Socrate ironise sur les orateurs et finit par répondre : « Crois-tu que tu serais toi-même capable de parler, s'il le fallait et que le Conseil t'eût choisi ? » (traduction EH). Platon a probablement été confronté personnellement à ce type de défi, surtout après avoir écrit le Gorgias, et l'oraison du Ménexène, si elle ne prouve pas sa capacité à écrire rapidement un tel discours, atteste par son existence même que l'on peut en écrire un sans être un orateur expérimenté, donc que ce type de discours ne relève pas d'un art véritable (DS, p. 18), contrairement à ce que prétendent souvent leurs auteurs (voir DS, p. 65 à propos de Lysias et EH, p. 96-97 sur Alcidamas). Comme le dit bien EH (p. 105), « l'oraison ne s'invente pas, elle se répète » selon Platon ( $c f$. DS, p. 29 : « the funeral oration is merely a mélange of clichés »). Ceci ne signifie cependant pas que la parodie et la critique qu'elle exprime soient purement techniques, elles sont également politiques dans la mesure où l'oraison mobilise sans distance des procédés oratoires, mais aussi des expériences et des valeurs communes, des stéréotypes et des récits quasi-légendaires. La description du régime politique athénien et les invocations de la « liberté » et de « l'égalité » par Socrate-Aspasie permettent vraisemblablement à Platon de mettre en relief, par une imitation à peine outrée, les faiblesses et même les contradictions qu'il percevait dans l'idéologie de l'Athènes démocratique, comme l'avait déjà bien vu Nicole Loraux et comme le confirment EH et DS sur de nombreux points, en particulier l'histoire des rapports entre Athènes et l'Empire perse ou bien la description de la manière dont la cité éduque les futurs citoyens.

Mais Ménexène est-il capable d'accéder à une telle critique des discours de sa cité ou ce point de vue est-il réservé à Socrate et au lecteur (idéal) du Ménexène ? EH (p. 12-13, 170-171) estime que les paroles de Ménexène dans le très court échange final (249d-e) peuvent être lues soit comme aussi naïves qu'au début, soit comme lucides à l'égard de l'oraison funèbre, et que le dialogue nous présente donc un Ménexène « ambivalent », ou plutôt, que sa trajectoire intellectuelle est ouverte et indécidable. DS (p. 25-26, 178-180) estime au contraire que Ménexène est entièrement admiratif de l'oraison de Socrate-Aspasie. Il s'agit là d'un des rares désaccords nets entre les lectures de DS et EH, qui adoptent pourtant des approches d'ensemble et de détail assez différentes, mais il est crucial. Si le Ménexène est assurément loin d'être le seul dialogue de Platon ayant donné lieu à des interprétations divergentes, les problèmes herméneutiques qu'il suscite font parfois songer aux paradoxes imaginés par Borgès dans sa fameuse nouvelle « Pierre Ménard, auteur du Quichotte », qui sont loin d'être atténués par l'absence de distance chronologique entre Platon et cette oraison funèbre athénienne dont il est et n'est pas l'auteur. 